\title{
THE MICROWAVE SPECTRUM OF SOLAR MILLISECOND SPIKES
}

\author{
M. Stähli and A. Magun \\ Institute of Applied Physics \\ University of Bern \\ Sidlerstrasse 5 \\ CH-3012 Bern, Switzerland
}

\begin{abstract}
The microwave radiation from solar flares sometimes shows short and intensive spikes which are superimposed on the burst continuum. New observations at $3.47 \mathrm{GHz}$ with high temporal and spectral resolution have revealed lifetimes of less than $10 \mathrm{~ms}$ and bandwidths between 0.5 and $200 \mathrm{MHz}$. Simultaneous measurements at two further frequencies showed no coincident spikes at the second and third harmonic. The spikes can be explained by the theory of electron cyclotron masering if the observed bandwidths are determined by magnetic field inhomogeneities or if the rise times are independent of the source diameters. The latter would imply source sizes between 50 and $100 \mathrm{~km}$.
\end{abstract}

\section{Introduction}

Milisecond microwave spikes are intensive and very short fine structures which are occasionally superimposed on the microwave continuum of solar flares (Figure 1). They have mainly been observed at the lower end of the microwave spectrum up to $\sim 6 \mathrm{GHz}$ (Dröge, 1977; Slottje, 1978 and 1980; Zhao, 1983; Stäh11 and Magun, 1985). The duration of most of the spikes was reported to be shorter than $20 \mathrm{~ms}$ and the deduced brightness temperatures of the spikes were in the range between $10^{13}$ and $10^{15} \mathrm{~K}$. In order to resolve and study the spectral and temporal fine structures of millisecond spikes a digital spectrometer with a time resolution of $5 \mathrm{~ms}$ was constructed (Stäh11, 1983). Its center frequency was set at $3.47 \mathrm{GHz}$ and its total bandwidth of $200 \mathrm{MHz}$ was continuous $1 \mathrm{y}$ covered by 30 channels with bandwidths varying between 0.5 $\mathrm{MHz}$ and $30 \mathrm{MHz}$. The frequency range was extended by two fixed frequency polarimeters at 3.1 and $5.205 \mathrm{GHz}$. For the simultaneous observation at the second and third harmonic of the gyrofrequency the latter was chosen at $3 / 2$ of the spectrometer center frequency. 
INSTITUTE OF APPLIED PHYSICS, UNIVERSITY OF BERNE, SHITZERLAND

I 3.2. F=3.2 GHz, $E W=100.0 \mathrm{MHZ}, T=0.100 \mathrm{SEC}$

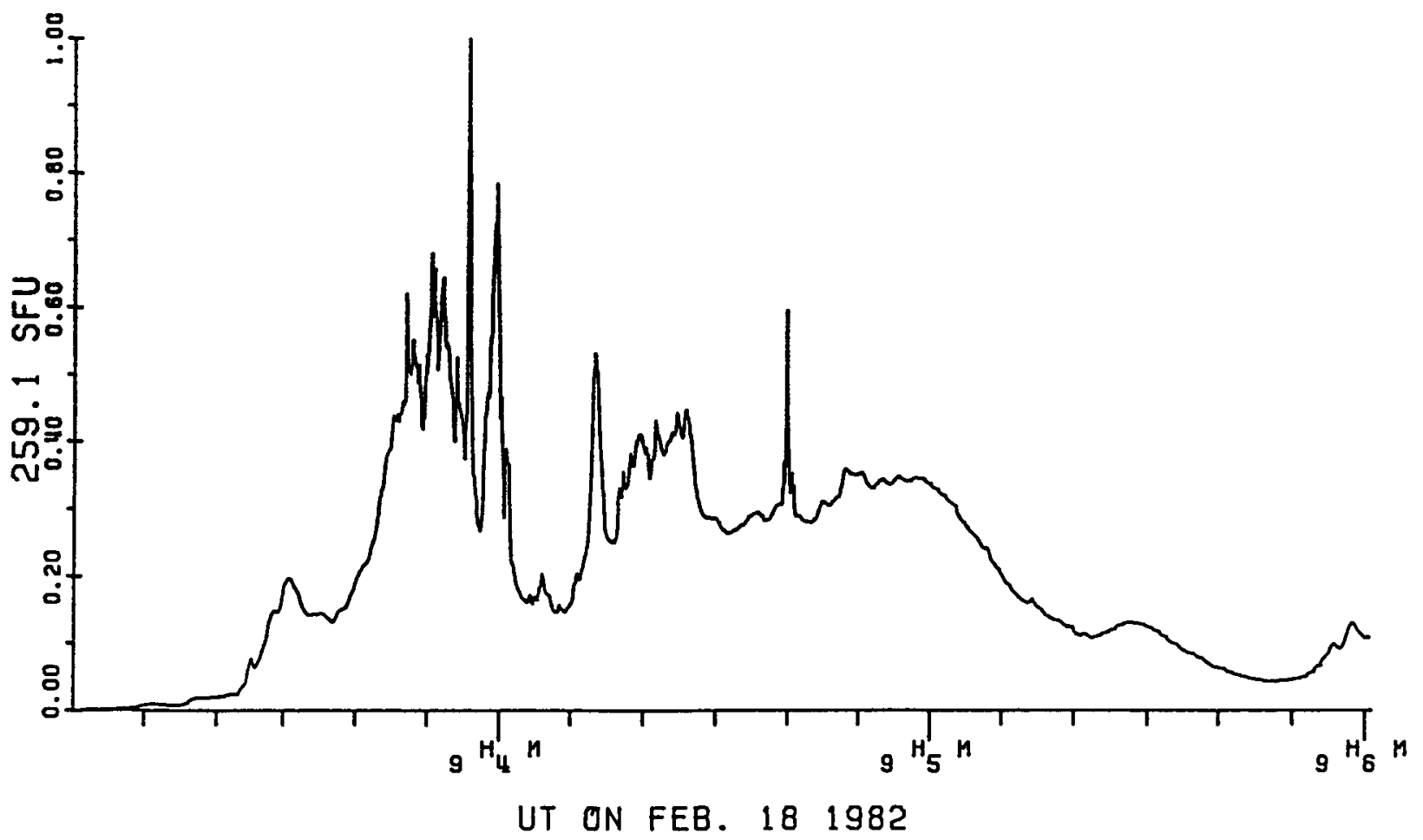

Fig. 1. Typical time profile of a solar radio burst at 3.2 GHz with superimposed millisecond spikes. The integration time $1 \mathrm{~s} 100 \mathrm{~ms}$.

In this paper new spectral observations of microwave spikes which were obtained with our fast spectrometer are presented and the proposed gyrosynchrotron maser emission tested. The concept of harmonic radiation by electron-cyclotron masers (Holman et al., 1980; Melrose and Dulk, 1982; Sharma et al., 1982) provides a plausible explanation for the observed characteristics of the spikes.

2. The spectrum of millisecond spikes

\subsection{Observations}

Between August 1982 and May 1983, 107 events have been observed, of which 14 exhibited microwave spikes. The fine structures appeared either as single spikes or in groups (Figure 2). The duration, rise and fall times of most of the spikes were between 5 and $10 \mathrm{~ms}$. These values correspond to the instrumental resolution of $5 \mathrm{~ms}$ and we therefore belleve that the temporal structures of these spikes could not be resolved. 


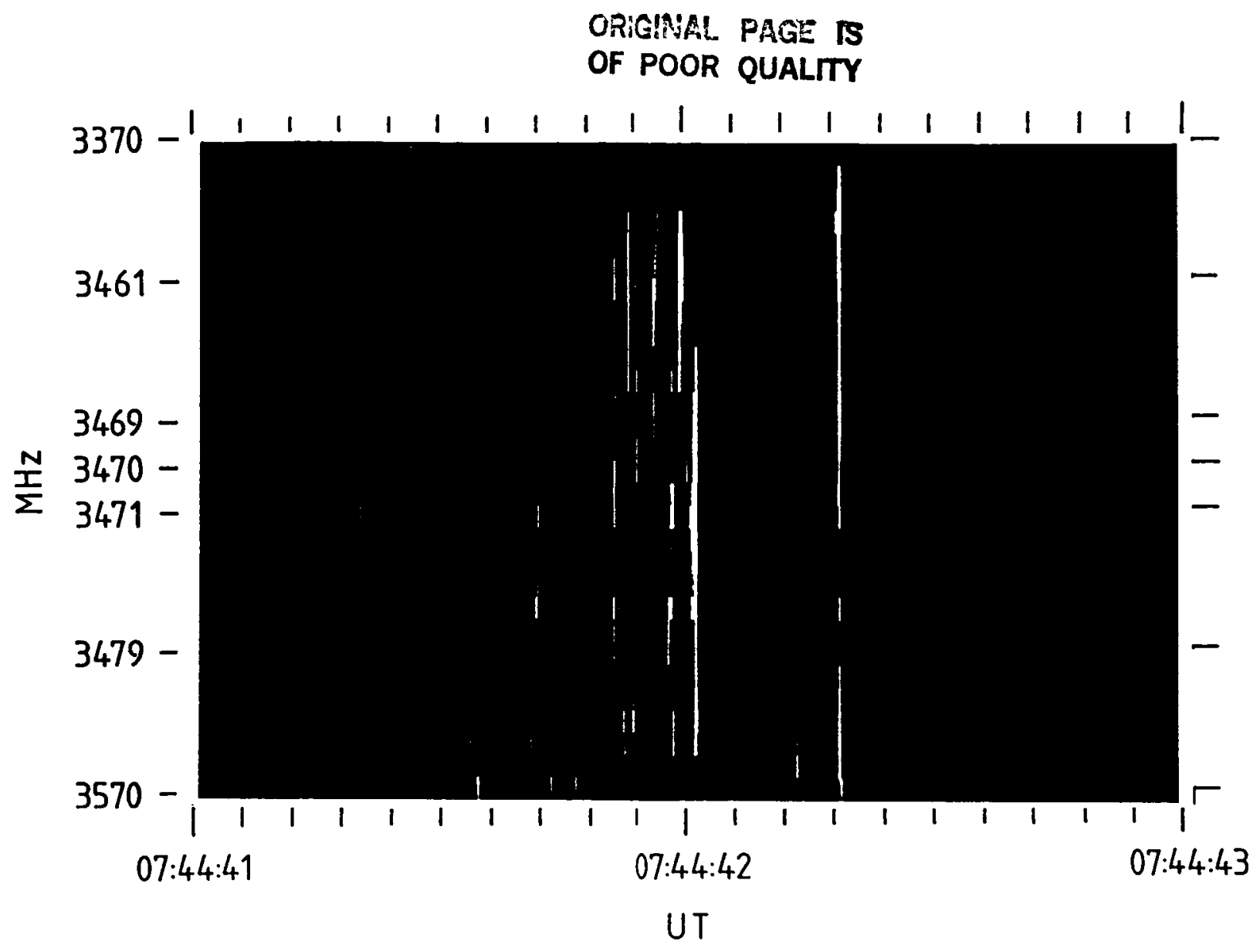

Fig. 2. Dynamic spectrum of millisecond spikes with subtracted continuum. The event of December 25, 1982 was observed with an integration time of 5 ms and a spectral resolution between 0.5 and $30 \mathrm{MHz}$.

The brightness temperatures of single spikes were derived from the rise time and the intensity under the assumption that the growing wave within the source propagates with the speed of light. Temperatures usually exceeded $10^{20}$ $\mathrm{K}$ but were always below $10^{12} \mathrm{~K}$. The bandwidth of the spikes varied from less than $0.5 \mathrm{MHz}$ to more than $200 \mathrm{MHz}$. However, the bandwidth must be smaller than approximately $300 \mathrm{MHz}$ because no simultaneous spikes have been observed with the spectrometer centered at $3.47 \mathrm{GHz}$ and the nearest single frequency polarimeter at $3.1 \mathrm{GHz}$. Additionally, no spikes were found to occur simultaneously at the second and third harmonic of the local gyrofrequency.

\subsection{Discussion}

The spectral data has been used to test the cyclotron maser theory (Melrose and Dulk, 1982). It predicts spike emission mainly slightly above the second or third harmonic of the local gyrofrequency with brightness temperatures up to $10^{25} \mathrm{~K}$ and very short rise times. The work of Melrose and 
Dulk (1982) was used to derive relationships between observed spike parameters as flux density, brightness temperature and bandwidth, which are suitable for testing the proposed emission mechanism. Because of the many free parameters describing the loss cone and the ambient plasma only approximate formulae were used.

The relationship between brightness temperature $T_{b}$ and relative bandwidth $\Delta w / w$ of the growing wave in the source was derived from (16) of Melrose and Du1k (1982):

$$
\mathrm{T}_{b} \nsim m v_{0}^{2}\left(\frac{2 \pi c}{\omega}\right)^{2} \frac{1}{\mathrm{~L} \mathrm{r}_{0}}
$$

where $v_{0}$ : mean velocity of the

m : electron mass radiating electrons

c: velocity of light $\quad \omega$ : frequency

$r_{0}$ : classical electron radius $L$ : trap length

With $\frac{\Delta w}{\omega} \approx v_{0}^{2} / c^{2}, \frac{\omega}{2 \pi} \approx 3 \mathrm{GHz}$ and $\mathrm{L} \approx 10^{10} \mathrm{~cm}$ this reduces to

$$
\mathrm{T}_{\mathrm{b}}[\mathrm{K}] \approx 10^{15} \frac{\Delta \omega}{\omega}
$$

which is a linear relationship between the brightness temperature and the relative bandwidth of the spikes under the assumption of a homogeneous source. The above expression is only valid if the saturation is due to the fundamental radiation which usually grows fastest. A recent and more detailed analysis of the growth rates (Winglee, 1985) has shown that for $\omega_{p} / \Omega_{2}>0.5$ the second harmonic dominates. This would lead to even higher brightness temperatures than given above.

The scatterplot (Figure $3 a$ ) shows the derived brightness temperatures and the relative bandwidths of the observed spikes, which were resolved in time and frequency. Relationship (1) is represented by a dashed 1ine. It is obvious that the two parameters are uncorrelated in contrast to the prediction. The deduced temperatures are too low or the relative bandwidths are too large for which several explanations are possible: 1) Larger observed bandwidths than predicted could be due to magnetic field inhomogeneities within the source whose influence is not included in the theory. 2) The low brightness temperatures could be the result of overestimated source sizes. In this case source diameters and observed rise times must be uncorrelated which is for instance true if the latter is determined by the dynamics of the electron distribution. 

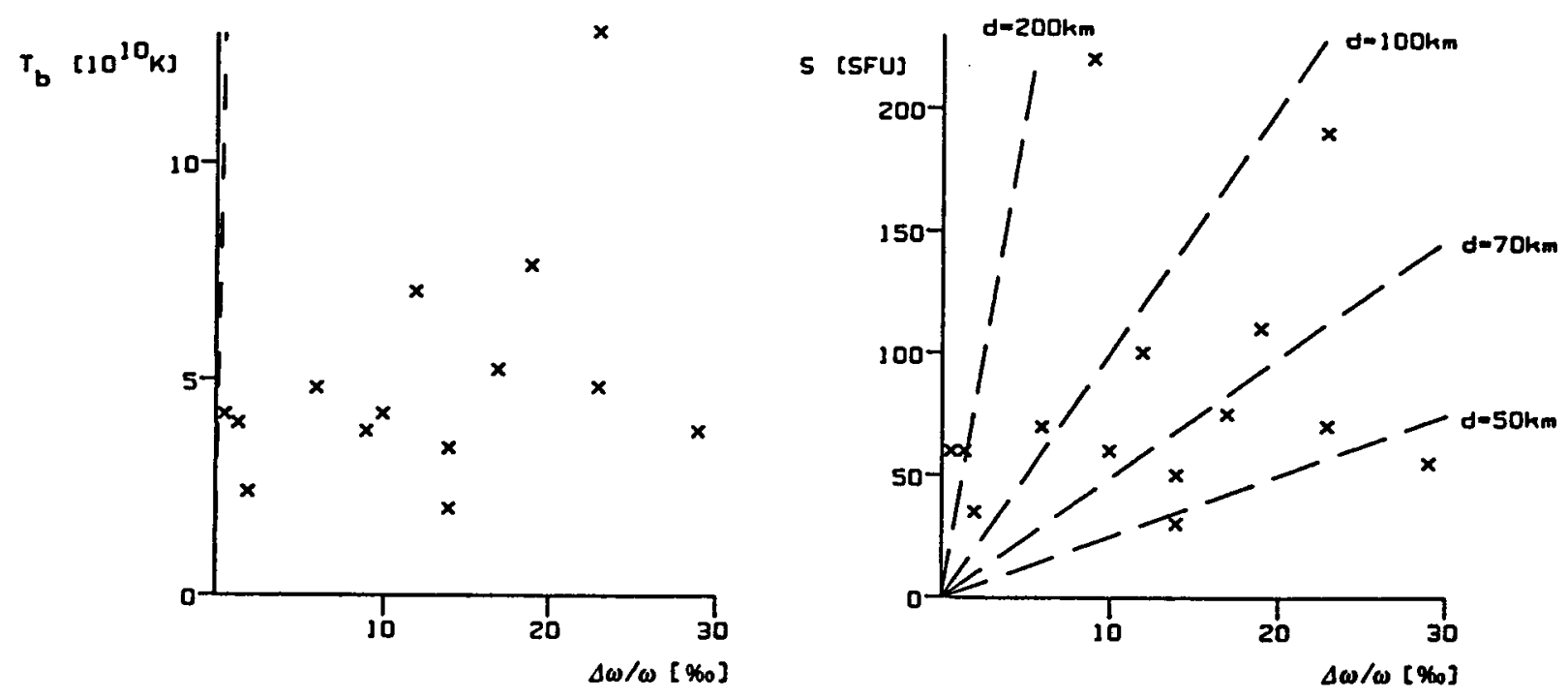

Fig. 3. Scatterplots of derived brightness temperature (a) and observed flux density (b) versus the relative bandwidth of observed spikes. The dashed lines represent the theoretical approximations. $d$ is the source diameter.

In order to avold the assumption of correlated source sizes and rise times the observed flux density of the same sample of spikes was plotted against the relative bandwidth (Figure $3 \mathrm{~b}$ ). The theoretical relationship for different source diameters was derived from (1) by using the Rayleigh-Jeans approximation:

$$
S[S F U] \approx d^{2}[\mathrm{~km}] \cdot \frac{\Delta w}{w}
$$

S : flux density in SFU $d$ : source diameter in $\mathrm{km}$

$\frac{\Delta w}{w}:$ relative bandwidth

If we assume that the bandwidth is not determined by inhomogeneities and the rise time does not depend on the source geometry, diameters between 50 and 100 $\mathrm{km}$ could explain most of the spikes.

These first observations of the spectral fine structures of millisecond spikes establish several new features. However, they also indicate that for a detailed analysis, better observations with higher temporal and spectral resolution including circular polarization are essential. For further investigation of the important electron spectrum it is also necessary to correlate microwave data with spike observations in other spectral ranges (hard $X$-ray, decimetric radio waves). 
Acknowledgements

The authors are indebted to Prof. E. Schanda for the opportunity to undertake this work. They would also like to thank Prof. G.A. Dulk for valuable discussions. The work was supported by the Swiss National Science Foundation under grant No. 2.604.0.82.

\section{References}

Dröge, F. 1977, Astron. Astrophys ., 57, 285.

Holman, G.D., Eichler, D. and Kundu, M.R. 1980, In M.R. Kundu and T.E. Gergely (eds.), 'Radio Physics of the Sun', IAU Symp., 86, 457.

Melrose, D.B. and Dulk, G.A. 1982, Astrophys. J., 259, 844.

Sharma, R.R., Vlahos, L. and Papadopoulos, K. 1982, Astron. Astrophys., 112, 377 .

Slottje, C. 1978, Nature, 275, 520.

Slottje, C. 1980, in M.R. Kundu and T.E. Gergely (eds.), 'Radio Physics of the Sun', IAU Symp., 86, 195.

Stäh11, M. 1983, 'Das Mikrowellen-Spektrum von solaren Millisekunden-Spikes', Diploma Thesis, Institute of Applied Physics, Bern.

Stähl1, M. and Magun, A. 1985, submitted to Solar Phys.

Winglee, R.M. 1985, submit ted to J. Geophys. Res.

Zhao, R. 1983, Adv. Space Res., 2, 177. 